Martina Wagner-Egelhaaf

\title{
Komposition und Aufführung.
}

\section{Louis Spohr's Selbstbiographie (1860/61)}

Die Literaturwissenschaft hat auf die Autobiographie eines Komponisten einen etwas anderen Blick als die Musikwissenschaft oder auch die Geschichtswissenschaft. Musikwissenschaftlerinnen oder Historiker lesen einen autobiographischen Text in erster Linie, weil sie etwas über das Leben des Autobiographen bzw. der Autobiographin erfahren und die Persönlichkeit kennenlernen wollen. Die Literaturwissenschaftlerin interessiert sich für die Form der Autobiographie als solche, die Entwicklung der Gattung, ihre Grenzen und Möglichkeiten. ${ }^{1}$ Es geht der literaturwissenschaftlichen Autobiographieforschung also nicht so sehr um die Person, den Autor, die Autobiographin, sondern um den Text als literarische Form und als Medium der Selbstkonstruktion. In der Literaturwissenschaft geht man davon aus, dass die Form eines Texts nicht lediglich einen Inhalt wiedergibt, sondern dass der Inhalt wesentlich durch die Form geprägt ist. D. h.: in literaturwissenschaftlicher Perspektive bildet sich in einer Selbstbiographie eine historische Person nicht einfach ab, sondern es wird davon ausgegangen, dass der Text mit seinen sprachlich-rhetorischen Mitteln ein autobiographisches Ich überhaupt erst hervorbringt und modelliert. Man darf den Louis Spohr im Text nicht mit dem historischen Louis Spohr gleichsetzen, allerdings kann man auch nicht behaupten, dass der Text-Spohr mit dem historischen Spohr nichts zu tun hat, denn letzterer hat ja schließlich den ersteren geschaffen. Der Text-Spohr ist gewissermaßen das Selbstbild des historischen Spohr und somit Teil seiner Lebensrealität. Und gerade dieses komplexe Wechselverhältnis, das heute auch unter dem Stichwort ,Autofiktion “ ${ }^{2}$ diskutiert wird, bildet den Untersuchungsgegenstand

\footnotetext{
${ }^{1}$ Über die Unterschiede literaturwissenschaftlicher und geschichtswissenschaftlicher Autobiographieforschung vgl. Volker Depkat, ,Zum Stand und zu den Perspektiven der Autobiographieforschung in der Geschichtswissenschaft“, in: BIOS. Zeitschrift für Biographieforschung, Oral History und Lebensverlaufsanalysen 23/2 (2010), S. 170-187; Martina Wagner-Egelhaaf, „Zum Stand und zu den Perspektiven der Autobiographieforschung in der Literaturwissenschaft", in: BIOS 23/2 (2010), S. 188-200.

${ }^{2}$ Vgl. Claudia Gronemann, „Autofiction“ und das Ich in der Signifikantenkette. Zur literarischen Konstitution des autobiographischen Subjekts bei Serge Doubrovsky“, in: Poetica 31 (1999), S. 237-261; dies., Postmoderne/Postkoloniale Konzepte der Autobiographie in der französischen und maghrebinischen Literatur. Autofiction - Nouvelle Autobiographie - Double Autobiographie - Aventure du texte, Hildesheim u.a. 2002; Frank Zipfel, „Autofiktion. Zwischen den Grenzen von Faktualität, Fiktionalität und Literarität?“, in: Grenzen der
} 
literaturwissenschaftlicher Autobiographieforschung. D.h. die Literaturwissenschaft interessiert sich mehr für das Selbstbild und seine mediale textuelle Form als für die außertextuelle Person des Autobiographen bzw. der Autobiographin.

Der oft bemühte Referenztext in der germanistischen Autobiographieforschung ist Goethes Dichtung und Wahrheit, das unter dem Obertitel Aus meinem Leben zwischen 1810 und 1833 publiziert wurde. Dichtung und Wahrheit wurde deswegen gleichsam zum Modell in der Autobiographieforschung, weil Goethes Lebensbericht eine Programmatik der Autobiographie formuliert. In der häufig zitierten Vorrede heißt es:

Denn dieses scheint die Hauptaufgabe der Biographie zu sein, den Menschen in seinen Zeitverhältnissen darzustellen, und zu zeigen, in wiefern ihm das Ganze widerstrebt, in wiefern es ihn begünstigt, wie er sich eine Welt- und Menschenansicht daraus gebildet, und wie er sie, wenn er Künstler, Dichter, Schriftsteller ist, wieder nach außen abgespiegelt. Hiezu wird aber ein kaum Erreichbares gefordert, daß nämlich das Individuum sich und sein Jahrhundert kenne, sich, in wiefern es unter allen Umständen dasselbe geblieben, das Jahrhundert, als welches sowohl den willigen als unwilligen mit sich fortreißt, bestimmt und bildet, dergestalt daß man wohl sagen kann, ein Jeder, nur zehn Jahre früher oder später geboren, dürfte, was seine eigene Bildung und die Wirkung nach außen betrifft, ein ganz anderer geworden sein. ${ }^{3}$

Hier wird, paradigmatisch für das 19. Jahrhundert, die hermeneutische Vorstellung des Individuums zum Ausdruck gebracht, das von seinen Zeitverhältnissen geprägt ist und diese zugleich beeinflusst. Man mag sich darüber wundern, dass Goethe seinen Lebensbericht ,Dichtung und Wahrheit' nennt, denn von einer Autobiographie erwartet man doch wohl, wenn schon nicht die Wahrheit, so doch in jedem Fall auch nicht ,Dichtung'. Aber Goethe war natürlich klug genug zu reflektieren, dass das autobiographische Ich in Bezug auf sich selbst nicht unbedingt der zuverlässigste Wahrheitsgarant ist. Und außerdem sah Goethe sehr klar, dass die ,Wahrheit‘ eines Lebens nicht in der Wiedergabe aller Fakten liegt, sondern dass ein Symbol dessen höhere Wahrheit oftmals sehr viel besser fassen kann. Und genau hier tritt die Dichtung in ihre Rechte. ${ }^{4}$

Literatur. Zu Begriff und Phänomen des Literarischen, hrsg. von Simone Winko, Fotis Jannidis und Gerhard Lauer, Berlin/New York 2009, S. 284-314; Auto(r)fiktion. Literarische Verfahren der Selbstkonstruktion, hrsg. von Martina Wagner-Egelhaaf, Bielefeld 2013.

${ }^{3}$ Johann Wolfgang Goethe, Aus meinem Leben. Dichtung und Wahrheit, in: Sämtliche Werke. Briefe, Tagebücher und Gespräche, Bd. 14, hrsg. von Klaus Detlef Müller (= 1. Abteilung: Sämtliche Werke 14), Frankfurt a. M. 1986, S. $13 \mathrm{f}$.

${ }^{4}$ Vgl. hierzu Martina Wagner-Egelhaaf, Autobiographie (=Sammlung Metzler 323), Stuttgart/Weimar ${ }^{2} 2005$, S. 2 f. 
Die beiden Bände von Louis Spohr's Selbstbiographie erschienen 1860 und $1861 .^{5}$ Angeregt dazu, sein Leben aufzuzeichnen, wurde Spohr durch den Kassler Publizisten Friedrich Oetker, der eine Schilderung der Feierlichkeiten anlässlich von Spohrs 25-jährigem Dienstjubiläum in Kassel vornahm und eine ausführliche Spohr-Biographie plante. Als er Spohr um die nötigen Unterlagen bat, beschloss dieser, die Niederschrift seines Lebens selbst in die Hand zu nehmen. Spohr kam damit zunächst bis zum Jahr 1822, also dem Beginn seiner Kasseler Zeit. Und er schrieb seinen Lebensbericht in der dritten Person, gleichsam als sein eigener Biograph. Als er nach einer fünfjährigen Arbeitspause im Frühjahr 1858 seine selbstbiographische Tätigkeit wieder aufnahm und sein Leben bis ins Jahr 1838 weiterschrieb, wechselte er in die erste Person und verfügte, dass bei einer späteren Herausgabe seiner Lebensaufzeichnungen alles in die IchForm überführt werden sollte. Nach Spohrs Tod machte sich seine zweite Frau, Marianne Spohr, geb. Pfeiffer, unterstützt von Spohrs Schwiegersohn, Johann Heinrich Wolff, an die Herausgabe der Selbstbiographie. Wolff verfasste auch ein Vorwort zum ersten Band. Für die Zeit nach 1838 wurde die Selbstbiographie in allererster Linie wohl von Marianne Spohr wieder in der Er-Form, also als Biographie, unter Verwendung von Briefen, privaten Aufzeichnungen, Konzertprogrammen und Zeitungsberichten, bis zum Tod des Komponisten fortgeführt. ${ }^{6}$ Der 1860/61 erschienene Text ist also ein Hybrid. Der Titel „Selbstbiographie“ wurde von den Herausgebern gewählt; Spohr selbst hat von seinen ,Lebenserinnerungen' gesprochen. Unter diesem Titel brachte Folker Göthel 1968 dann auch eine kritische Ausgabe heraus, die sich um die Herstellung eines authentischen Texts bemüht und deshalb nur die Zeit bis 1822 umfasst, die Spohr tatsächlich selbst beschrieben hat. Schon für den Berichtszeitraum von 1822 bis 1838 waren, wie auch eine Fußnote in der Selbstbiographie festhält, starke redigierende Eingriffe der Herausgeber erfolgt. ${ }^{7}$ Göthel, der Spohrs Nachlass und auch das Originalmanuskript der Lebenserinnerungen eingesehen hat, kommt zu dem Urteil, „daß es sich bei dem bisher allen bekannten Druck der sogenannten ,Selbstbiographie“ von 1860/61 um eine von den Vorlagen in vieler Hinsicht abweichende Bearbeitung handelt. ${ }^{* 8}$ Wenn den folgenden Ausführungen dennoch die Erstausgabe zugrundeliegt und die Edition von Göthel nur korrigierend herangezogen wird, so deshalb, weil sie ein kulturgeschichtliches Zeugnis darstellt, das ein Bild von Spohr vermittelt

\footnotetext{
${ }^{5}$ Louis Spohr's Selbstbiographie, 2 Bde., Kassel/Göttingen 1860-1861 (künftig zitiert als Sb).

${ }^{6}$ Vgl. dazu Folker Göthel, „Vorwort“, in: Louis Spohr, Lebenserinnerungen. Erstmals ungekürzt nach den autographen Aufzeichnungen hrsg. von Folker Göthel, Tutzing 1968, S. IX-XX.

${ }^{7}$ Vgl. Sb, Bd. 2, S. 156.

${ }^{8}$ Göthel, ,Vorwort", S. XIII.
} 
wie es ein Jahrhundert lang rezipiert wurde und in dem Selbst- und Fremdbilder in ein sich gegenseitig beleuchtendes, konstruktives Wechselverhältnis treten.

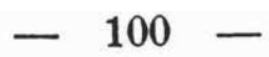

mals ben 3 uhborern bei ihrem cintritt in ben Saal überreidht wurbe, geidentt hat. (58 lautet:

NEW ARGYLL ROOMS.

MI. SPPIII'S CONCERT.

Thursday, June 18 1820.

P A R T I.

Grand Sinfonia (M. S.) . . . . . . . Spohr.

Air, Mr. T. W elch ${ }_{n}$ Revenge, revenge, Thimotheus cries" ". Ha endel.

Grand Duetto (M. S.), Harp and Violin, Mad. Spohr and Mr. Spohr. . . . . Spohr.

Aria, Miss Goodall „una voce al cor mi parla." Clarinet obligato Mr. Willman . . P $\mathbf{P} r$.

Sestetto for Pianoforte, two Violins, Viola, Violoncello and Contrabasso, Messrs.: Rie s, Watts, Wagstaff, R. Ashley, Lindley and Dragonetti . . . Ries.

Irish Melodies (M. S:) with Variations for the Violin, Mr. Spohr (composed expressly for this occasion)........ Spohr. P A R T II.

Nonetto for Violin, Viola, Violoncello, Contrabasso, Flute, Oboe, Clarinet, Horn and Bassoon, Messrs. Spohr, Lindley, Drag on et ti, Ireland, Griesbach, Willman, Arnull and Holmes. . . . . . Spohr.

Scena, Mrs. Salmon Fellon, la pena arrai" . Rossini.

Rondo for the Violin, Mr. Spohr . . . . Spohr.

Aria, Mr. V a ugh a n ${ }_{\eta}$ Rendi'l sereno" . . . Ha end el.

Overture

Leader of the Band.$^{*}$. Mr. Spohr.

At the Pianoforte. . Sir George Smart.

Die neue, vom Draefter nun idon getannte, aber bods nod)= mals jorgfältig burdaprobirte Symphonie murbe meifterbaft ere= cuttixt und fand too möglid nod Yebhafteren Beifall, alz bei ber

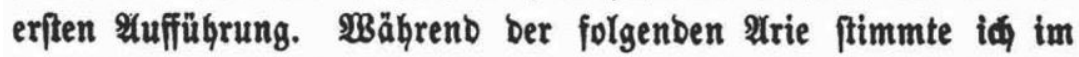

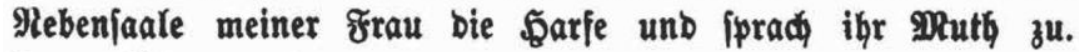

Abbildung 1: Ausschnitt aus Louis Spohr's Selbstbiographie (Bd. 2, S. 100)

Hier zeigen sich schon bemerkenswerte Unterschiede zum autobiographischen Modellfall von Goethes Dichtung und Wahrheit. Goethe, der Literat, stellt dezidiert die eigene Individualität in 
den Mittelpunkt, die in ihrer Eingebundenheit in ihre Zeit für die Leserschaft einen Bildungswert beanspruchte. ${ }^{9}$ Der Autor von Dichtung und Wahrheit wird gleichsam zum Schöpfer seiner selbst als literarisches Kunstwerk. Louis Spohr's Selbstbiographie hat indessen mehrere Autoren und sie ist wohl auch eher das, was man heute eine Gebrauchsautobiographie nennen würde, die keinen künstlerischen Gestaltungsanspruch erhebt. Dass aber gerade auch hier natürlich kulturelle Muster, Topoi und Techniken der Selbstdarstellung zum Einsatz kommen, macht einen solchen sich eher unkünstlerisch gebenden Text $\mathrm{zu}$ einem sprechenden kulturgeschichtlichen Zeugnis. Nun beschäftigt sich die literaturwissenschaftliche Autobiographieforschung vornehmlich mit der literarischen Autobiographie, mit Texten also, die einen Kunstanspruch haben. Hingegen stellen sicherlich populäre Autobiographien bzw. Autobiographien von Persönlichkeiten des öffentlichen Lebens aus Politik, Wirtschaft, Kultur, Sport und Showbusiness das quantitativ größere Segment auf dem Buchmarkt dar. Diese Autobiographien sind in der Regel sehr viel weniger literarisch ambitioniert und sie folgen mehr oder weniger unkritisch den Konventionen der Gattung, während die literarische Autobiographie häufig damit spielt und versucht, die Gattungsgrenzen zu durchbrechen bzw. zu erweitern. Und doch bildet eben die ,nichtliterarische“ Autobiographie, wie sie der Einfachheit halber genannt sei, den Kernbestand des Genres, eben die Autobiographie, die ganz naiv einfach Einblick in gelebtes Leben geben möchte. Und dass dabei der berufliche Tätigkeitsbereich des Autobiographen bzw. der Autobiographin eine zentrale Rolle spielt, liegt auf der Hand. Ja, der Beruf gehört sogar gattungshistorisch $\mathrm{zu}$ den begründenden Motiven der Lebensbeschreibung. So nennt der Autobiographieforscher Günter Niggl die Berufsautobiographie als zweite gattungsprägende Traditionslinie neben der religiösen Bekenntnisautobiographie. ${ }^{10}$ Und eine solche

\footnotetext{
${ }^{9}$ Der fingierte Brief eines Freundes, den Goethe im Vorwort zu Dichtung und Wahrheit zur Begründung seines autobiographischen Projekts zitiert, reklamiert, dass die Reflexion des Schriftstellers auf das von ihm Hervorgebrachte „denen abermals zur Bildung gereiche, die sich früher mit und an dem Künstler gebildet haben“ (Goethe, Dichtung und Wahrheit, S. 12). Demgegenüber hält auch Göthel fest: „Wenn es nicht überhaupt Spohrs Veranlagung widersprach, zu einer Selbstbetrachtung im tiefern Sinne zu gelangen, so war es zumindest nicht seine Art, andern gegenüber davon Zeugnis abzulegen, das eigene Leben aus den Perspektiven seiner Zeit zu erkennen, Ursprung und Richtung seiner Entwicklung aufzudecken und zu begründen“ (Göthel, „Vorwort“, S. XIVf.). Dies scheint direkt gegen das Goethe'sche Modell gerichtet.

${ }^{10}$ Günter Niggl, ,Zur Säkularisation der pietistischen Autobiographie im 18. Jahrhundert“, in: Die Autobiographie. Zu Form und Geschichte einer literarischen Gattung, hrsg. von Günter Niggl, Darmstadt ${ }^{2} 1998$, S. 366-391, hier S. 372: „Die Tradition der religiösen Konfession will nach augustinischem Vorbild die Bekehrung als den Angelpunkt des eigenen Lebens darstellen und dieses von Anfang an auf jenes Hauptereignis hinordnen; sie wird durchkreuzt von der Tradition der Berufs-(meist Gelehrten-)Autobiographie, die den beruflichen Werdegang von den Studienjahren in die öffentliche Wirksamkeit mit ihren wechselnden Erfolgen und Konflikten als das eigentliche autobiographische Thema betrachtet und seit dem Ende des 16. Jahrhunderts durch mehrere umfangreiche, zunächst
} 
Berufsautobiographie, in der die berufliche Tätigkeit des Autobiographen im Zentrum steht, ist ganz zweifellos die Spohr'sche. Im Text läuft alles schnurstracks auf den Violinvirtuosen und späteren Komponisten hin. Und das ist natürlich auch konsequent. So fragt etwa Christa Wolf in ihrem autobiographischen Roman Kindheitsmuster von 1976: „Wie sind wir so geworden, wie wir heute sind?"11 und formuliert damit ein grundlegendes Erkenntnismotiv der Autobiographie, das gleichsam ein linear-progressives Gewordensein des autobiographischen Ichs voraussetzt. Und entsprechend liest man gleich auf den ersten zwei Seiten von Louis Spohr's Selbstbiographie das Folgende:

Die Eltern waren musikalisch; der Vater blies Flöte, die Mutter, Schülerin des Kapellmeisters Schwaneberger in Braunschweig, spielte sehr fertig das Clavier und sang die italienischen Bravourarien der damaligen Zeit. Da sie sehr oft des Abends musicirten, so wurde der Sinn und die Liebe zur Tonkunst schon früh bei mir geweckt. So begann ich, mit einer klaren Sopranstimme begabt, zu singen, und im vierten oder fünften Lebensjahre schon, durfte ich in Duetten mit der Mutter an den Abendmusiken Theil nehmen. Um diese Zeit war es, daß mir der Vater, meinem dringend geäußerten Wunsche nachgebend, auf einem Jahrmarkte eine Geige kaufte, auf der ich nun unaufhörlich spielte. Zuerst versuchte ich, die früher gesungenen Melodien herauszubringen und war überglücklich, wenn die Mutter dazu accompagnierte. ${ }^{12}$

Dann erfährt man die Einzelheiten von Spohrs musikalischer Ausbildung, ${ }^{13}$ dem ersten Geigenunterricht bei dem Rektor Riemenschneider, dann ab 1790/91 bei Herrn Dufour, einem französischen Emigranten. In dieser Zeit beginnt der kleine Louis auch schon zu komponieren:

Während des Unterrichts bei Herrn Dufour machte ich auch meine ersten Compositionsversuche, bevor ich noch irgend einen Unterricht in der Harmonie erhalten hatte. Es waren Duette für zwei Violinen, die ich mit meinem Lehrer in den Abendmusikpartien vortrug und damit die Eltern im höchsten Grade überraschte. Noch erinnere ich mich des stolzen Gefühls, nun auch als Componist vor den Freunden des Hauses auftreten zu können. Als Honorar erhielt ich von den Eltern einen Prachtanzug, bestehend in einer rothen Jacke mit Stahlknöpfen und einem gelben Beinkleid nebst Schnürstiefeln mit Quasten, w[o]rum ich schon lange, wiewohl vergebens, sollicitirt hatte. $^{14}$

noch lateinisch geschriebene Werke, vor allem in Frankreich (Junius, Thuanus, Huetius), aber auch in Deutschland (Ursinus, Andreä) vertreten ist und im 18. Jahrhundert eine neue Blütezeit erleben wird.“"

${ }^{11}$ Christa Wolf, Kindheitsmuster. Roman, Darmstadt und Neuwied: Luchterhand, 1979, S. 397.

${ }^{12} \mathrm{Sb}, \mathrm{Bd} .1$, S. $1 \mathrm{f}$.

${ }^{13}$ Spohr weist darauf hin, dass er in jungen Jahren viel zeichnete und malte und eine Zeitlang schwankte, ,welche der beiden Künste, Musik oder Malerei, [er] als Lebensberuf erwählen wolle“. Sb, Bd. 1, S. 16.

${ }^{14} \mathrm{Sb}$, Bd. 1, S. 3. 
Damit sind die Hauptmotive von Spohrs Lebenserzählung gesetzt: Komposition und Aufführung. Der insgesamt linear-chronologisch verfahrende Lebensbericht informiert darüber, wann und unter welchen biographischen Umständen Spohr seine Werke komponierte und wann, wo, wie und mit welchem Erfolg sie aufgeführt wurden. Komposition und Aufführung sind zweifellos die Leitmotive dieses Lebens. Dabei fällt auf, dass der Vorgang der Komposition kaum vertieft oder gar problematisiert wird. Da gibt es keine Introspektion oder ein Ringen des Genies. Unzufrieden ist Spohr nur mit den ersten Opernversuchen und Quartetten ${ }^{15}$ und gegen Ende seines Lebens, als es mit dem Komponieren nicht mehr so richtig klappt. ${ }^{16}$ Ansonsten läuft das Komponieren aber wie am Schnürchen. Fast mehr Aufmerksamkeit als dem schöpferischen Akt der Komposition widmet der Selbstbiograph dem Aspekt der Aufführung. Genauso positiv überrascht und begeistert wie das frühe elterliche Publikum reagiert auch die spätere Zuhörerschaft der Spohr'schen Aufführungen. ${ }^{17}$ Da werden sehr genau der Rahmen der Aufführung und die Reaktionen der in der Regel begeisterten Zuhörer und Zuhörerinnen beschrieben. Zur Dokumentation der Aufführungserfolge, und vielleicht auch um sich nicht selbst permanent loben zu müssen, werden häufig Zeitungsberichte herangezogen und wörtlich wiedergegeben. So wird z. B. die Musikzeitung zu einem in Dresden im Herbst 1807 gegebenen Konzert mit den folgenden Worten zitiert:

„Der dritte (unter den fremden Concertgebenden) war der rühmlichst bekannte HerzoglichSachsen-Gothaische Concertmeister, Herr Spohr, der sich auf der Violine, sowie seine Frau auf der Pedalharfe, hören ließ. Nicht sobald wird wieder ein Künstler Ursache haben, so vollkommen mit der Aufnahme zufrieden zu sein, die ihm hier zu Theil ward, als Herr Spohr, und gewiß wird jeder Freund der Kunst laut eingestehen, daß er diese Auszeichnung reichlich verdiente. ${ }^{118}$

Die Stichwörter Komposition und Aufführung bilden nicht nur die Leitmotive in Louis Spohr's Selbstbiographie, sondern sie stellen darüber hinaus auch geeignete Kategorien dar, um die

\footnotetext{
${ }^{15}$ Vgl. ebd., Bd. 1, S. 125 und 130.

${ }^{16}$ Vgl. ebd., Bd. 1, S. 130 und Bd. 2, S. 379.

${ }^{17}$ Zum ausführlich gewürdigten Komplex der Aufführung gehört auch das von Spohr eingeführte Dirigieren mit dem Taktstock, über dessen positive Resonanz er nicht ohne Stolz berichtet: „Durch diesen Erfolg überrascht und begeistert, gab das [Londoner] Orchester auch sogleich nach dem ersten Satze der Symphonie seine allgemeine Billigung der neuen Direktionsweise laut zu erkennen und beseitigte dadurch alle weitere Opposition von Seiten der Direktoren“. Sb, Bd. 2, S. 87.

${ }^{18} \mathrm{Sb}, \mathrm{Bd} .1, \mathrm{~S} .110 \mathrm{f}$.
} 
Verfasstheit dieser Autobiographie in den Blick zu nehmen. Von ihrer zusammengesetzten „Komposition', d.h. ihrer mehrstimmigen Autorschaft, war bereits die Rede. Die Vorrede des Schwiegersohns, die ein Charakterbild von Louis Spohr als besonders liebenswürdiger, bescheidener Persönlichkeit entwirft ${ }^{19}$, stellt eine Art Ouvertüre zu dieser Lebensaufführung dar und das von Marianne Spohr gezeichnete Lebensbild der Jahre von 1838 bis zum Tod Spohrs ist ein enthusiastisches Finale, das die monumentalen Erfolge des Meisters und die ihm allerorts entgegengebrachten Huldigungen in den Vordergrund stellt. Unter kompositionellen Gesichtspunkten ist des Weiteren festzuhalten, dass Spohr wie auch seine späteren Herausgeber nicht nur Kritiken, Konzertankündigungen und Briefe in den Text integriert, sondern immer wieder auf sein Tagebuch zurückgreift und ausführlich aus diesem zitiert. Das Tagebuch, das viel Anekdotisches enthält, aber über weite Strecken auch von einem eher uninspirierten aufzählenden Duktus bestimmt ist, stellte sicherlich eine wichtige Gedächtnishilfe für die Komposition der Lebensbeschreibung dar, widerspricht aber dem von Philippe Lejeune formulierten autobiographietheoretischen Kriterium, dass die Autobiographie eine „[r]ückblickende Prosaerzählung“ ist und Nachdruck auf die Geschichte der Persönlichkeit legt. ${ }^{20}$ Im hermeneutischen Verständnis der Autobiographie verbindet sich mit dem geschichtlichen Aspekt der Gedanke vom Lebenszusammenhang und der Entwicklung, ja der ,Bildung' im doppelten Wortsinn des autobiographischen Ichs. ${ }^{21}$ Das Tagebuch hingegen beschreibt aus der Situation heraus und hat keinen Entwicklungszusammenhang im Blick. ${ }^{22}$ Der Herausgeber der Lebenserinnerungen Folker Göthel moniert denn auch die Uneinheitlichkeit in

\footnotetext{
${ }^{19} \mathrm{Vgl}$. Sb, Bd. 1, S. vii. Dort liest man auch etwa: „Spohr war, wie alle edlen Naturen, streng sittlich und von einer fast mädchenhaften Züchtigkeit.“ (Sb, Bd. 1, S. xii) und gleich darauf „Spohr zeigt sich überall muthvoll, entschlossen, tapfer, mit einem Worte echt männlich“ (Sb, Bd. 1, S. xiii).

${ }^{20}$ Philippe Lejeune, Der autobiographische Pakt, aus dem Französischen von Wolfram Bayer und Dieter Horning, Frankfurt a. M. 1994, S. 14.

${ }^{21}$ In der eingangs zitierten Passage aus Goethes Dichtung und Wahrheit, ist der Bildungsgedanke zentral (vgl. Goethe, Dichtung und Wahrheit, S. 13; vgl auch S. 12). Vgl. auch Wilhelm Dilthey, Der Aufbau der geschichtlichen Welt in den Geisteswissenschaften, Einleitung von Manfred Riedel, Frankfurt a. M. 1981, S. 25f.: „Die Selbstbiographie ist nur die zu schriftstellerischem Ausdruck gebrachte Selbstbesinnung des Menschen über seinen Lebensverlauf. [...] Nur sie macht geschichtliches Sehen möglich. [...] Zu den allgemeinen Kategorien des Denkens traten im Verstehen des Lebens die von Wert, Zweck und Bedeutung hinzu. Unter diesen standen dann umfassende Begriffe wie Gestaltung und Entwicklung des Lebens. [...] Indem wir zurückblicken in der Erinnerung, erfassen wir den Zusammenhang der abgelaufenen Glieder des Lebensverlaufs unter der Kategorie ihrer Bedeutung." Die hermeneutische Konzeptualisierung der Autobiographie stellt in der literaturwissenschaftlichen Autobiographieforschung eine theoretische Basisreferenz dar, von der sich spätere Konzepte, etwa poststrukturalistische oder feministische, abgrenzen.

${ }_{22}$ Zum Tagebuch vgl. Peter Boerner, Tagebuch, Stuttgart 1969; Manfred Jurgensen, Das fiktionale Ich. Untersuchungen zum Tagebuch, Bern, 1979.
} 
der sprachlichen Gestaltung der Selbstbiographie. ${ }^{23}$ Man könnte hier aber auch - und dies passt in das historistische 19. Jahrhundert - ein dokumentarisches Bewusstsein der eigenen Historizität erkennen. In welcher Weise sich Spohr zu seinem Tagebuch verhält bzw. Gebrauch von ihm macht, exemplifziert die folgende Sequenz:

Nach der Ankunft in Leipzig am 29. November gibt das Tagebuch noch zwei kurze Berichte und verstummt dann gänzlich. Der erste bespricht eine Aufführung der Oper von Paer: „die Wegelagerer“. Der zweite erzählt von einem Besuche des GewandhausConcertes.

„Diese Concerte“, heißt es, „werden von einer Gesellschaft von Kaufleuten veranstaltet. Es sind aber keine Dilettanten-Concerte $[\ldots]^{6 \cdot 24}$

Tatsächlich bringen die Tagebuchaufzeichnungen, die zumeist unter dem frischen Eindruck des Erlebten stehen, einen - die musikalische Metapher sei erlaubt - anderen Ton in den Text, der gerade nicht einheitlich komponiert ist wie der Goethe'sche, sondern sich, um einmal mehr in der Sprache der Musik zu bleiben, durch eine gewisse Vielstimmigkeit ${ }^{25}$ auszeichnet. In diesem Sinn lässt sich auch die Kategorie der ,Aufführung“ in einem spezifischen Sinn auf den Text der Spohr'schen Selbstbiographie beziehen. Wenn nämlich nicht nur eine Person spricht, die des Autobiographen, sondern auch dessen früheres alter ego des Tagebuchschreibers sowie Briefpartner und Musikkritiker zu Wort kommen, entsteht ein textueller Raum, der den Porträtierten aus verschiedenen Perspektiven beleuchtet. Eine weitere Funktion der Zeitdokumente liegt in der szenischen Vergegenwärtigung der Vergangenheit, die den Zusammenhang des Lebensverlaufs in den Hintergrund treten lässt. Tatsächlich muss man sich in der Lektüre wiederholt zeitlich orientieren, wenn mit dem Wechsel der Genres auch zeitliche Sprünge einhergehen. ${ }^{26}$

Spohrs Selbstbiographie setzt sich also aus unterschiedlichen autobiographischen Genres zusammen. Neben der rückblickenden Erzählung, die es natürlich auch gibt, dem Gestus des Tagebuchs und des Briefs weist der Text Züge auf, die man eher dem Genre der Memoiren als dem der Autobiographie zuschreibt. Bernd Neumann hat bereits in den 1970er-Jahren die Unterschiede zwischen Autobiographie und Memoiren idealtypisch herausgestellt. Die

\footnotetext{
${ }^{23}$ Vgl. Göthel, ,Vorwort“, XV.

${ }^{24} \mathrm{Sb}, \mathrm{Bd} .1, \mathrm{~S} .78$.

${ }^{25}$ Besonders anschaulich beispielsweise Sb, Bd. 1, S. 47.

${ }^{26}$ Vgl. etwa Sb, Bd. 2, S. 50, wo der Autobiograph bei der Lektüre seiner Tagebuchaufzeichnungen Dinge vermisst, die er nun beginnt nachzutragen.
} 
Autobiographie stellt Neumann zufolge eher die Gattung des innengeleiteten Individuums dar, das die Geschichte seiner Entwicklung bis zur Eingliederung in die Gesellschaft schildert. In der bürgerlichen Gesellschaft ist dieser Zeitpunkt in der Regel mit dem Eintritt in das Berufsleben und der Eheschließung gegeben. Memoiren, so Neumann, sind eher das Genre des außengeleiteten, Traditionen und Bräuchen verhafteten Individuums, das seine berufliche Stellung erreicht hat und aus dieser heraus Zeit und Mitmenschen beobachtet. ${ }^{27}$ Tatsächlich ist Spohr's Selbstbiographie voll von sehr lebendigen Schilderungen von Begegnungen mit unterschiedlichen Zeitgenossen, Schülern, fürstlichen Dienstherren, Dichtern ${ }^{28}$, aber insbesondere natürlich anderen Musikern und Komponisten, denen Spohr ein lebhaftes Interesse entgegenbringt, sowohl ihrer Person gegenüber als auch ihrem Werk und der Art und Weise ihrer Kunstausübung. So finden sich z. T. anrührende Porträts von Beethoven ${ }^{29}$, Mendelssohn ${ }^{30}$, Wagner $^{31}$, Paganini ${ }^{32}$ und anderen musikalischen Größen der Zeit. Auch politische und kulturelle Verhältnisse an den unterschiedlichen Orten von Spohrs Wirken und insbesondere seiner ausgedehnten Reisen werden mit Teilnahme geschildert. Ein memoirentypischer Zug ist der Hang zum Anekdotischen, der den Text streckenweise sehr unterhaltsam macht. So erzählt der Autobiograph etwa von einem Konzert, das ein reicher Altonaer Musikfreund im Jahr 1811 organisiert hatte. Vor dem Konzert lud er die Mitwirkenden, einschließlich des Ehepaars Spohr, zu einem luxuriösen Essen ein, bei dem so reichlich dem Champagner zugesprochen wurde, dass man das Konzert darüber völlig vergaß und erst, als das Publikum sich in Erinnerung brachte, in den Konzertsaal eilte. Mit viel Sinn für Situationskomik berichtet Spohr von einem mehr als leicht derangierten Auftritt:

\footnotetext{
${ }^{27}$ Vgl. Bernd Neumann, Identität und Rollenzwang. Zur Theorie der Autobiographie, Frankfurt a. M. 1970; vgl. auch die überarbeitete und erweiterte Neuauflage Bernd Neumann, Von Augustinus zu Facebook. Zu Geschichte und Theorie der Autobiographie, Würzburg 2013 (zum Verhältnis von Autobiographie und Memoiren ebd. S. 79-119).

${ }^{28} 1807$ zählen auch Goethe und Wieland in Weimar zu Spohrs und seiner Frau Dorettes Publikum: „Unter den Zuhörern im Hofconcerte befanden sich auch die beiden Dichter-Heroen Goethe und Wieland. Letzterer schien von den Vorträgen des Künstlerpaares ganz hingerissen zu sein und äußerte dies in seiner lebhaft-freundlichen Weise. Auch Goethe richtete mit vornehm-kalter Miene einige lobende Worte an uns" (Sb, Bd. 1, S. 109). Zu Goethe und Wieland vgl. auch Bd. 1, S. 125. Im Jahr 1818 ist Jean Paul unter Spohrs Zuhörern in Frankfurt (vgl. Bd. 2, S. 60). Auch Begegnungen mit Immermann und Grabbe (vgl. Bd. 2, S. 203) sowie mit Alexander von Humboldt, Ludwig Tieck, Friedrich Carl von Savigny (vgl. Bd. 2, S. 298) finden statt.

${ }^{29} \mathrm{Vgl}$. insbesondere Sb, Bd. 1, S. 197-203.

${ }^{30}$ Vgl. ebd., Bd. 2, S. 306-308.

${ }^{31}$ Vgl. ebd., Bd. 2, S. 271 und 305.

${ }^{32}$ Vgl. ebd., Bd. 1, S. 299 und Bd. 2, S. 180.
} 
[...] Man brach nun eiligst nach dem Concertsaale auf; doch war eigentlich Niemand mehr in der gehörigen Verfassung, um öffentlich auftreten zu können. Auffallend war dabei, daß die sonst Zaghaften nun die Muthigsten geworden waren. Das Altonaer DilettantenOrchester, dem die Hamburger Künstler als Kern und Stütze dienen sollten, war schon aufgestellt, und das Concert begann daher sogleich mit einer Ouvertüre von Romberg ${ }^{33}$, die er selbst leitete. Er, dem man nicht mit Unrecht vorwarf, daß er die Tempi seiner Compositionen stets zu langsam nehme, übereilte das Allegro seiner Ouvertüre diesmal dermaßen, daß die armen Dilettanten gar nicht mitkommen konnten. Es fehlte daher nicht viel, so wäre schon in der Ouvertüre umgeworfen worden. Nun folgten wir, meine Frau und ich, mit einer Sonate für Harfe und Violine, die wir, wie immer, ohne Noten vortragen wollten. Als wir schon saßen, und ich eben zu beginnen dachte, flüsterte mir meine Frau, die sonst die Besonnenheit selbst war, ängstlich zu: „Um des Himmelswillen, Louis, ich kann mich nicht besinnen, welche Sonate wir spielen wollen, und wie sie anfängt!" Ich sang ihr den Anfang heimlich in's Ohr und brachte sie so wieder zu der nöthigen Ruhe und Besonnenheit. [...]

Am schlimmsten erging es aber dem armen Schwenke ${ }^{34}$. Ihm hatte das Diner die Hosenschnalle gesprengt, ohne daß er es bemerkt hatte. Als er nun bei einem Potpourri mit Quartettbegleitung, das ich zum Schluß des Concertes spielte, zur Uebernahme der Violapartie auf die Erhöhung des Orchesters getreten war, fühlte er bald nach Beginn der Musik, daß ihm durch die Bewegung der Bogenführung das Beinkleid zu sinken begann. Viel zu gewissenhafter Musiker, um von seinen Noten etwas auszulassen, wartete er ganz ruhig die Pausen ab, um das Beinkleid wieder heraufzuziehen. Seine Noth blieb dem Publikum nicht lange verborgen und erregte große Heiterkeit. Als ihn nun aber am Ende des Potpourris eine Sechzehntel-Bewegung dermaßen schüttelte, daß das Sinken des Beinkleids bedenkliche Fortschritte machte und ans Unanständige zu streifen drohte, da konnte das Publikum sich nicht mehr halten und brach in allgemeines Kichern aus. ${ }^{35}$

Genauso anschaulich schildert Spohr seine ausgedehnten Reisen. Der Reisebericht ist ein weiteres autobiographisches Register, das die Spohr'sche Selbstbiographie maßgeblich bestimmt. Die Reise als Motiv und Gegenstand der Beschreibung ist in der autobiographischen Tradition häufig ein Bild für die Lebensreise ${ }^{36}$ und von daher schon strukturell autobiographisch kodiert. Dies ist z. B. ganz deutlich der Fall bei Goethes Italienischer Reise, die Goethe explizit als Zweite Abteilung von Aus meinem Leben in den Jahren 1816 und 1817 veröffentlichte und in der er u.a. seine ,Wiedergeburt ${ }^{*}$ in Rom beschreibt. ${ }^{37}$ Auch Goethes Italienische Reise ist als

\footnotetext{
${ }^{33}$ Andreas Romberg (1767-1821), Cousin des Cellisten Bernhard Romberg (1767-1841), Geiger und Komponist, vertonte u.a. Schillers „Lied von der Glocke“.

${ }^{34}$ Christian Friedrich Gottlieb Schwencke (1767-1822), Komponist, Pianist, von 1788-1822 Kirchenmusikdirektor in Hamburg. Zur Freundschaft Spohrs mit Romberg und Schwenke vgl. Clive Brown, Louis Spohr. A Critical Biography, Cambridge, London u.a. 1984, S. 60.

${ }^{35} \mathrm{Sb}$, Bd. 1, S. 166-168.

${ }^{36} \mathrm{Vgl}$. Matthias Christen, to the end of the line. Zu Formgeschichte und Semantik der Lebensreise, München 1999.

${ }^{37}$ Goethe entfloh dem Druck der Weimarer Amtsgeschäfte und wollte sich in Italien als Künstler, evtl. sogar als Maler und Zeichner, neu erfinden. Letztlich feierte er denn aber doch seine Wiedergeburt als Dichter. Vgl. Johann
} 
Tagebuch angelegt, als ein Tagebuch, das für die Veröffentlichung gehörig überarbeitet wurde. Während Goethe vor allem die Werke der bildenden Kunst in Italien beschreibt, berichtet Spohr vordringlich - und zumeist sehr kritisch ${ }^{38}$ - über das italienische Musikleben. Und wie Goethe 1787 (mehrfach) den Vesuv besteigt so auch Spohr dreißig Jahre später. ${ }^{39}$ Beide wagen sich, entgegen der Warnung ihrer touristischen Führer, bis zum Kraterrand vor. Und natürlich findet genau dann ein kleiner Ausbruch statt, so dass den verwegenen Reisenden Lava und Asche um die Ohren fliegen.

Wir waren aber kaum ein paar hundert Schritte gegangen, so warf der eine der Krater mit einem fürchterlichen Gekrach eine ungeheuere Menge glühender Steine aus, von denen einige nicht allzuweit von uns niederfielen. [...] Nach vieler Mühe sahen wir uns indessen doch endlich oben und standen nun auf dem schmalen Rande des Kraters, der wie ein Trichter, etwa zweihundert Fuß an der oberen Oeffnung im Durchmesser, gestaltet ist. Nachdem wir einige Minuten hier verweilt und den Ausbrüchen des anderen Kraters, der unter'm Winde vor uns lag, zugesehen hatten, wurde der, bei welchem wir standen, plötzlich ganz von Rauch befreit, und wir konnten nun in die grause Tiefe hinabschauen. Da sahen wir in dem Grunde des Trichters zwischen Felsenmassen große Schlünde, aus denen die Flammen hervorbrachen; doch da gleich wieder Rauch darauf folgte, so war dieser Blick nur von kurzer Dauer. Einer der Engländer bekam sogar Lust, in einem Augenblick, wo der Rauch von dem Krater, auf dessen Rande wir standen, nicht stark war, auch zu dem anderen hinüber zu laufen, um einen Blick in dessen Tiefe zu werfen. Er hatte aber kaum den Rand erreicht, so erfolgte ein glücklicherweise nicht sehr starker Ausbruch, vor dem er kaum noch Zeit genug hatte, sich wieder zu uns zu retten. In demselben Augenblicke fing auch ein dritter Krater hinter uns an zu lärmen, und nun war es hohe Zeit, uns aus dem Staube zu machen. Jener warf zwar nur Asche aus, wurde aber durch den Schrecken, den er uns eingejagt hatte, unser Erretter vom völligen Untergange; denn kaum waren wir wieder auf unserem alten Lagerplatze, so warf der bis jetzt sehr ruhige Krater, an dessen Rande wir gewesen waren, eine solche Menge glühender Steine aus, und zwar gerade nach der Seite hin, wo wir gestanden hatten, daß wir sämmtlich erschlagen und verschüttet worden wären, hätten wir uns noch fünf Minuten dort oben verweilt. Nachdem sich die verwegene Gesellschaft von ihrem starren Schrecken erholt hatte, mußten wir unseren Vorwitz eingestehen, trotz der Warnung der Führer uns so weit hinaufgewagt zu haben. $^{40}$

Wolfgang Goethe, Italienische Reise. Teil 1, in: Sämtliche Werke. Briefe, Tagebücher und Gespräche, Bd. 15/1, hrsg. von Christoph Michel und Hans-Georg Dewitz (= 1. Abteilung: Sämtliche Werke 15/1), Frankfurt a. M. 1993, S. $158-160,556$.

${ }^{38}$ Italien ist ihm ein „Sibirien der Kunst“ (Sb, Bd. 1, S. 294, vgl. auch Bd. 1, S. 296, 322f., 337und Bd. 2, S. 38). In seinen Urteilen ist Spohr bisweilen recht drastisch, auch wenn er sich zu der Aussage versteigt, „daß die Franzosen ein unmusikalisches Volk sind“" (Sb, Bd. 2, S. 122; vgl. Bd. 2, S. 124, 136 und 139).

${ }^{39}$ Vgl. Sb, Bd. 2, S. 3-7.

${ }^{40} \mathrm{Sb}$, Bd. 2, S. 5f. Bei Goethe liest sich der Vulkan-Besuch folgendermaßen: „Noch klapperten die kleinen Steine um uns herum, noch rieselte die Asche, als der rüstige Jüngling mich schon über das glühende Gerille hinaufriß. Hier standen wir an dem ungeheuren Rachen, dessen Rauch eine leiste Luft von uns ablenkte, aber zugleich das 
Das Erlebnis des Vulkanausbruchs gehörte offensichtlich zum touristischen Gruselprogramm und zum Topos der Italienreise. Dass Autobiographien in hohem Maße topisch und eben gar nicht so individuell sind, hat Stefan Goldmann herausgearbeitet. ${ }^{41}$ Man könnte sogar sagen, dass die Individualität des Lebensberichts gerade einer der klassischen Topoi der Autobiographik darstellt. ${ }^{42}$ Das sich Der-Todesgefahr-Aussetzen und das Überstehen der Lebensgefährdung hat im autobiographiereflexiven Kontext nicht nur Unterhaltungswert, sondern kann zudem als Bestätigung des Lebensverlaufs und als Bekräftigung des Weiterlebens gelesen werden. Wenn im Folgenden dann vom Rückzug der Kraterschauer an einen sichereren Platz die Rede ist und Spohr berichtet, dass „,[m]ehrere aus der Gesellschaft die Bemerkung [machten], daß es doch eine wahre Thorheit sei, sein Leben so dem Ungefähr anzuvertrauen, um eine eitle Neugierde zu befriedigen“" ${ }^{43}$, ist ein alter autobiographischer Topos aufgerufen, derjenige nämlich der Besteigung des Mont Ventoux durch Francesco Petrarca im Jahr 1336. Auf dem Gipfel angelangt, greift Petrarca bekanntlich in Anbetracht der sich unter ihm ausbreitenden Landschaft einer plötzlichen Eingebung folgend zu der Taschenausgabe von Augustinus' Confessiones, die er stets bei sich führt, um dort auf die in der Folge vielzitierten Worte zu stoßen: „,Und es gehen die Menschen, zu bestaunen die Gipfel der Berge und die ungeheuren Fluten des Meeres und die weit dahinfließenden Ströme und den Saum des Ozeans und die Kreisbahnen der Gestirne und haben nicht acht ihrer selbst. ““44 Spohr indessen bricht bewusst oder unbewusst diese traditionsmächtige Anspielung ironisch um, wenn er anfügt: „Diese Betrachtungen hinderten indessen nicht, daß wir die mitgebrachten Eier, welche die Führer zu unseren Füßen in der

Innere des Schlundes verhüllte, der ringsum aus Tausend Ritzen dampfte. Durch einen Zwischenraum des Qualmes erblickte man hie und da geborstene Felsenwände. Der Anblick war weder unterrichtend noch erfreulich, aber eben deswegen weil man nichts sah verweilte man um etwas heraus zu sehen. Das ruhige Zählen war versäumt, wir standen auf einem scharfen Rande vor dem ungeheuren Abgrund. Auf einmal erscholl der Donner, die furchtbare Ladung flog an uns vorbei, wir duckten uns unwillkürlich, als wenn uns das vor den niederstürzenden Massen gerettet hätte; die kleineren Steine klapperten schon, und wir, ohne zu bedenken daß wir abermals eine Pause vor uns hatten, froh die Gefahr überstanden zu haben, kamen mit der noch rieselnden Asche am Fuße des Kegels an, Hüte und Schultern genugsam eingeäschert“" (Goethe, Italienische Reise, S. 210; vgl. S. 203f. und 209f.).

${ }^{41}$ Vgl. Stefan Goldmann, „Topos und Erinnerung. Rahmenbedingungen der Autobiographie“, in: Der ganze Mensch. Anthropologie und Literatur im 18. Jahrhundert, hrsg. von Jürgen Schings, Stuttgart und Weimar 1994, S. 660-675; ders., Christoph Wilhelm Hufeland im Goethekreis. Eine psychoanalytische Studie zur Autobiographie und ihrer Topik, Stuttgart 1993.

${ }^{42}$ Vgl. Gabriele Schabacher, Topik der Referenz. Theorie der Autobiographie, die Funktion, Gattung' und Roland Barthes ' Über mich selbst, Würzburg 2007.

${ }^{43} \mathrm{Sb}, \mathrm{Bd}$. 2, S. 6.

${ }^{44}$ Francesco Petrarca, „An Francesco Dionigi von Borgo San Sepolcro in Paris“, in: Francesco Petrarca, Dichtungen, Briefe, Schriften, hrsg. von Hanns W. Eppelheimer. Frankfurt a. M. 1980. S. 89-98, hier S. 96. 
heißen Asche kochten, mit vielem Appetit verzehrten und uns dazu einen Trunk Lacrymae Christi trefflich schmecken ließen“. ${ }^{45}$

Dass Spohrs künstlerisches Ausdrucksmittel die Musik war und eben nicht das geschriebene Wort, zeigt sich in der Selbstbiographie nicht zuletzt in der Einschaltung zahlreicher Notenbeispiele $^{46}$, die zum einen die intensive Auseinandersetzung des Autors mit seinen musikalischen Gegenständen dokumentieren und die autobiographischen Aufzeichnungen etwa des Tagebuchs auch zum Arbeitsdokument machen, zum anderen aber eine rhetorische Funktion im Hinblick auf die Leserinnen und Leser der Selbstbiographie erfüllen: Um ihnen die Gedanken und Probleme zu vermitteln, die ihn umtrieben, bedurfte es eines anderen Zeichensystems als der Buchstaben. Hier kommt dem Notenbild naheliegenderweise die größere Evidenz zu. Namentlich die Auseinandersetzung mit der fremden und von Spohr vielfach als mängelbehaftet befundenen Musik Italiens machte den Wechsel in das andere Notationssystem erforderlich. ${ }^{47}$

\footnotetext{
${ }^{45} \mathrm{Sb}$, Bd. 2, S. 6.

${ }^{46} \mathrm{Vgl}$. Sb, Bd. 1, S. 50, 207f., 257, 307-309, 317, 330f., 334 und 338-341; Bd. 2, S. 28f., 38, 106 und 117.

${ }^{47}$ Eine nicht untalentierte graphische Verzierung und einen Sachkommentar hat ein/e Leser/in des 2. Bandes von Spohr's Selbstbiographie im digitalisierten Exemplar der Bayrischen Staatsbibliothek hinterlassen. Spohr gibt eine Äußerung des Direktors am Mailänder Konservatorium Zingarelli zu Mozart wieder: ,ja, auch dieser sei nicht ohne Anlagen gewesen, er habe nur zu kurze Zeit gelebt, um sie gehörig ausbilden zu können; wenn er noch zehn Jahre fortstudirt hätte, so würde er wohl einmal etwas Gutes haben schreiben können“" (Sb, Bd. 2, S. 18f.).
} 


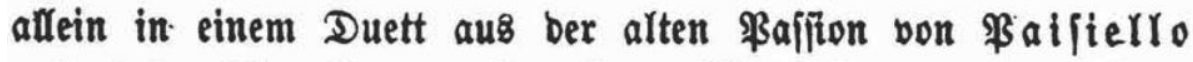
auf einige Secunben gerührt hat. Ebbenfalls neu, von $\mathfrak{o f =}$ fi ni zuerft gebraucht, ift auch bie 2 rt, wie er bie parlanten Steflen in ber Opera buffa, bie man bisker getwöhnlich auf einen Ton,

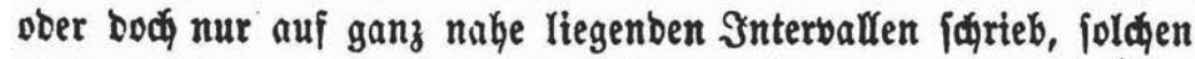
Bejangftêlfen unterlegt, bie man bis jekgt nur legato vorzutragen pflegte, wie z. B. im 2 nfange bezి obigen Duettę:
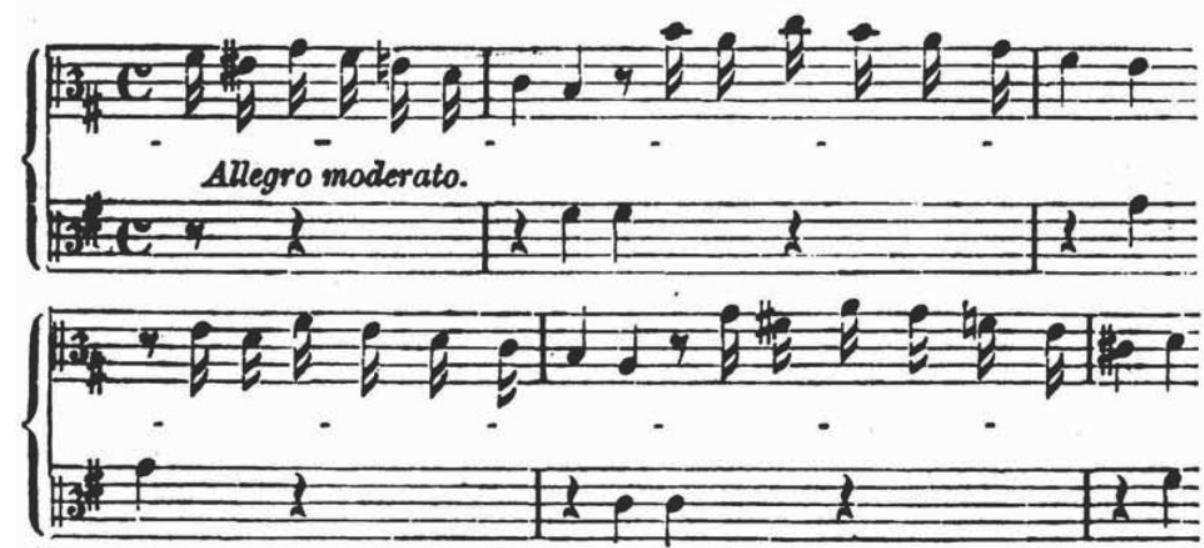

So betannt aud biejer 2 Anfang ift (er ähnelt bem 2 anfang einez Finale in eincm $\mathfrak{1} a \not b n$ 'iden Quartett aus Es-dur):

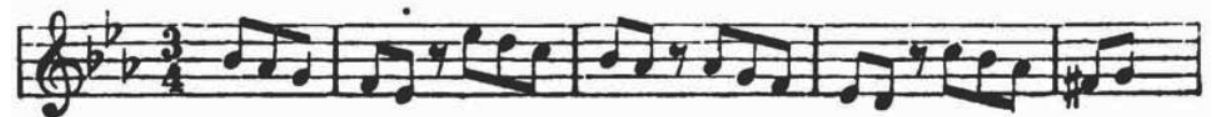

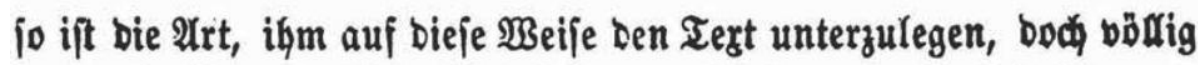
neu; ob aber gut, ift nod bie frage; mir tlang er wenigftę immer wie traveftirt, als wenn man z. B. auf einem fingenden $\Im n$ ftrumente einen Bejang, ber einen gefüblvollen Bortrag erlaubt, zum Sałerze io taritirt vorträgt, Daß er Rađen ftatt Rührung erregt. $\mathfrak{B}_{e}=$ nigftens würbe fein Эnjtrumentalift von Bsejdmad ben obigen Bejang staccato vortragen.

Abbildung 2: Ausschnitt aus Louis Spohr's Selbstbiographie (Bd. 1, S. 339)

Was die Oratorien anbelangt, so nehmen sie in der zweiten Hälfte des zweiten Bands von Louis Spohr's Lebensbeschreibung vergleichsweise viel Raum ein. Spohr äußert sich beispielsweise ausführlich zur Komposition und den diversen Aufführungen seines Oratoriums Die letzten 
Dinge. Zur Erstaufführung zitiert er einen Brief vom 26. März 1826, der einen anschaulichen Eindruck von der Art und Weise gibt, wie kalkuliert die Aufführung inszeniert wurde:

Der gestrige Tag war für die hiesigen Musikfreunde ein sehr festlicher; denn eine so solenne Musik-Aufführung, wie die meines Oratoriums, hat in Cassel noch nicht stattgehabt. Sie war Abends bei beleuchteter Kirche. Mein Schwiegersohn Wolff, der lange in Rom war, machte den Vorschlag, die Kirche wie in Rom am Charfreitage, durch Kreuzbeleuchtung zu erhellen und führte auch diese Idee aus. Ein vierzehn Fuß langes, mit Silberfolie überklebtes und mit 600 Glaslampen behängtes Kreuz, schwebte in der Mitte der Kirche und verbreitete ein so helles Licht, daß man allenthalben die Textbücher lesen konnte. Das Orchester- und Sängerpersonal, beinahe 200 Personen stark, war auf der oberen Emporkirche terrassenförmig aufgestellt und für die Zuhörer größtentheils unsichtbar. Das aus etwa 2000 Personen bestehende Auditorium beobachtete eine feierliche Stille. Meine beiden Töchter, die Sänger Wild, Albert und Föppel und noch ein Dilettant, sangen die Soli, und die Aufführung war fehlerlos. Die Wirkung war, wie ich mir selbst sagen mußte, außerordentlich. ${ }^{48}$

Im wohl vorwiegend aus der Feder von Marianne Spohr stammenden (um nochmals die musikalische Metaphorik zu bemühen) „Finale“ von Louis Spohr's Selbstbiographie, das als regelrechter Triumphzug Spohrs durch Europa angelegt ist, ${ }^{49}$ stellen die Oratorien einen passenden Rahmen für die Verklärung des Meisters. ${ }^{50}$ Das Publikum der europäischen Konzertsäle liegt Spohr zu Füßen und überschüttet ihn mit Huldigungen. ${ }^{51}$ Die Selbstbiographie zitiert den Monthly Chronicle, der zur Aufführung des Oratoriums Des Heilands letzte Stunden in Norwich schreibt: „Man kann mit Recht von diesem Oratorium sagen, daß ein göttlicher Hauch es durchweht; mehr als irgend ein Werk der neueren Zeit ist es aus warmem Herzen hervorgequollen und kann nicht ohne Thränen gehört werden.... ““. ${ }^{52}$ Und der Norwich Mercury

\footnotetext{
${ }^{48} \mathrm{Sb}$, Bd. 2, S. 171.

${ }^{49} \mathrm{Vgl}$. Sb, Bd. 2, S. 397.

${ }^{50}$ Wenn es im Hinblick auf die Fortsetzung der Selbstbiographie durch die Mitglieder von Spohrs Familie heißt, man wolle „ohne irgend welche schriftstellerische Gewandtheit zu beanspruchen, in einfach schmuckloser Wahrheitstreue, nach Spohr's eignem Beispiel, seine Lebensbeschreibung zu Ende [...] führen“ (Sb, Bd. 2, S. 224), ist zum einen ein Topos des autobiographischen Schreibens aufgerufen, der der Wahrhaftigkeit (vgl. WagnerEgelhaaf, Autobiographie, S. 41-43), zum anderen das Inszenierungspotenzial der Schreibenden allzu klein geredet. Was freilich gegen Ende der Selbstbiographie ebenfalls herausgestellt wird, ist Spohrs politische Unterstützung der deutschen liberalen Nationalbewegung, die ihn sogar in die Frankfurter Nationalversammlung führte (vgl. Sb, Bd. 2, S. 323-329).

${ }^{51}$ Vgl. Sb, Bd. 2, S. 282, 287f., 292-295, 297f., 313, 317f., 320, 330f. und 334f.

${ }^{52}$ Ebd., Bd. 2, S. 239.
} 
beschreibt das Verklingen des letzten Akkords mit den Worten: „Es war ein Moment heiliger Empfindung [...] der Eindruck war zu überwältigend und ließ alles Irdische verschwinden“.53

Quartett und Chor Nr. 16 aus dem zweiten Teil seines Oratoriums Die letzten Dinge, das Spohr im Juli 1859 nochmals in Würzburg gehört hatte, wurden denn auch bei Spohrs Beisetzung am 25. Oktober 1859 gespielt und so legt Marianne Spohr ihren Mann auch in der Selbstbiographie mit den folgenden Worten zur letzten Ruhe, mit denen die Aufführung von Spohrs Leben endet:

Die frommen Töne, die einst in heiliger Begeisterung seiner reinen Seele entquollen - denen er selbst noch vor wenig Wochen in stiller Andacht gelauscht, - sie klangen trauernd nun an seinem Grabe, und schmerzvoll, doch tröstlich zugleich hallt‘ es nah und fern in allen Herzen wieder:

„Selig sind die Todten, die in dem Herrn sterben, von nun an in Ewigkeit. Sie ruhen von ihrer Arbeit, und ihre Werke folgen ihnen nach!“ (II, 407) ${ }^{54}$

${ }^{53}$ Ebd., Bd. 2, S. 245.

${ }^{54}$ Ebd., Bd. 2, S. 407. 